\title{
Niveles de plomo en sangre en niños de 8 a 10 años y su relación con la alteración en el sistema visomotor y del equilibrio
}

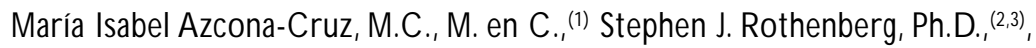 \\ Lourdes Schnaas-Arrieta, M. en Psic., ${ }^{(4)}$, Manuel Romero-Placeres, M.C., M. en $C .{ }^{(1,5)}$ \\ Estela Perroni-Hernández, Psic. ${ }^{(4)}$
}

\begin{abstract}
Azcona-Cruz MI, Rothenberg SJ, Schnaas-Arrieta L, Romero-Placeres M, Perroni-Hernández E. Niveles de plomo en sangre en niños de 8 a 10 años y su relación con la alteración en el sistema visomotor y del equilibrio. Salud Publica Mex 2000;42:279-287.
\end{abstract}

\section{Resumen}

Objetivo. Identificar si existe una asociación negativa entre los niveles de plomo en sangre y la coordinación visomotora y el equilibrio de escolares. Material y métodos. Se realizó un estudio transversal en 255 niños de 8 a 10 años de edad, que asistían a escuelas del Sector 1 del Instituto de Educación Pública del Estado de 0 axaca, durante noviembre y diciembre de 1998 . Se les aplicaron las pruebas de evaluación de la percepción visual de Frostig y la subescala de equilibrio Frostig Movement Skills Test Battery y se les tomó una muestra de sangre para la determinación de plomo por medio de espectrometría de abso rción atómica. Asimismo, se aplicó un cuestionario sociodemográfico y se evaluó la historia de salud del niño. Se relacionó el nivel de plomo sanguíneo con los resultados de las pruebas de percepción visual y del equilibrio mediante modelos de regresión múltiple. Se usó la técnica de simulaciones de Montecarlo para probar el efecto del plomo dentro del modelo. Resultados La media geométrica de las concentraciones de plomo en sangre fue de $11.5 \mu \mathrm{g} / \mathrm{dl}$ (DE geométrica $=+6.3,-5.2)$. El nivel de plomo se relacionó significativamente con el cociente de integración visomotora ( $p>0.042$ ). Este disminuye 1.78 (IC 95\%-3.51,-0.06) puntos por cada incremento de $10 \mu \mathrm{g} / \mathrm{dl}$ en los niveles de plomo

\author{
Azcona-Cruz MI, Rothenberg SJ, Schnaas-Arrieta L, \\ Romero-Placeres M, Perroni-Hernández E. \\ Relationship of blood lead levels \\ with visual-motor and equilibrium disturbances \\ in children aged 8 to 10 years. \\ Salud Publica Mex 2000;42:279-287.
}

\begin{abstract}
A bstract
Objective To assess the association between blood lead concentrations and visual-motor coordination and equilibrium in school age children. Material and methods In N ovember-D ecember 1998, a cross-sectional study was conducted among 255 children aged 8-10, who attended public schools in Sector 1 of the 0 axaca State Public Education Institute. Data were collected using the Frostig Evaluation of Visual Perception test and the equilibrium subscale of the Frostig Movement Skills Test Battery. A blood sample was taken to measure lead levels by atomic absorption spectrometry. Socioeconomic data and health histories were collected for use as control variables. Statistical analysis consisted of multiple regression models to test the relationship between blood lead level and the visual-motor and equilibrium tests.W e assessed the efect of lead within the model using 1000 Montecarlo simulations. Results. The geometric mean of blood lead concentrations was $11.5 \mu \mathrm{g} / \mathrm{dl}$ (geometric standard deviation $+6.3,-5.2$ ). After adjusting for control variables, the visual-motor integration subscale was significantly related to blood lead concentration $(p>0.042)$.The visual-motor integration value decreased $1.78(95 \% \mathrm{Cl}-3.51,-0.06)$ points for each $10 \mu \mathrm{g} / \mathrm{dl}$ increase in blood lead concentration.A mong the fo ur sub-tests com-
\end{abstract}

Este estudio fue realizado gracias a un financiamiento otorgado por la 0 rganización Panamericana de la Salud.

(1) Instituto N acional de Salud Pública (IN SP), México.

(2) Centro de Investigación en Salud Poblacional, IN SP, México.

(3) D epartment of A nesthesiology, Center for Environmental Research, Charles R. D rew University of Medicine and Science, Los Angeles, CA, United States of America.

(4) Instituto $\mathrm{N}$ acional de Perinatología. México, D.F., México.

(5) Instituto $\mathrm{N}$ acional de Higiene, Epidemiología y Microbiología de Cuba, La Habana, Cuba.

Fecha de recibido: 17 de noviembre de 1999 - Fecha de aceptado: 23 de junio de 2000

Solicitud de sobretiros: Stephen J. Rothenberg. Center for Environmental Research, Charles R. D rew University of Medicine and Science. Los Angeles (CA) 90059, United States of America.

Correo electrónico: drlead@gte.net 
en sangre del niño. De las pruebas que constituyeron este cociente, sólo se relacionó significativamente el plomo con la de coordinación ojo-mano $(p=0.045)$ y de relaciones espaciales $(p=0.039)$. El ingreso familiar también se relacionó significativamente con el cociente de integración visomotora, a mayor ingreso los resultados en las pruebas del niño fueron más altos. Sólo $3.1 \%$ de los niños obtuvieron puntuaciones consideradas clínicamente anormales. $\mathrm{N}$ o se encontró una relación significativa entre el plomo y el cociente de la respuesta motriz reducida, el cociente de percepción visual general y las pruebas de equilibrio. Conclusiones La relación negativa entre el plomo y la habilidad visomotora concuerda con los resultados encontrados en niños en otros países. Al parecer las concentraciones de plomo en sangre, que son comunes en los niños de 0 axaca, son suficientes para ocasionar una limitación visomotora de naturaleza sutil. Los resultados de este estudio señalan la necesidad de reforzar la iniciativa de reducir la exposición de los niños a las fuentes de exposición conocidas, en especial barro vidriado y, por lo tanto, reducir los niveles poblacionales de plomo en sangre.

Palabras clave: plomo/sangre; desempeño psicomotor; equilibrio; niño; México prising the visual-motor integration subscale, only eye-hand coordination $(p=0.045)$ and spatial relations $(p=0.039)$ were significantly related to blood lead. The visual-motor integration subscale was also significantly related to family income; greater income was related to greater testing scores. 0 nly $3.1 \%$ of the children had clinically abnormal testing scores. N o statistically significant association was found between blood lead levels and the Frostig subscale Index of Reduced Motor Response, the General Visual Perception Index, or any other equilibrium tests. Conclusions. The inverse relationship between blood lead concentration and visual-motor skill is consistent with results from studies in other countries. Blood lead levels common among children of 0 axaca are sufficient to produce subtle visualmotor impairments. These findings point out the need to strengthen the initiative to reduce child exposure to known lead sources, particularly lead-glazed ceramics, and thus lower the blood lead levels of the population.

Key words: lead/blood; psychomotor performance; equilibrium; child; Mexico
S e ha documentado que el plomo puede ocasionar $S$ daño a concentraciones bajas y que los niños son más sensibles que los adultos a sus efectos. ${ }^{1}$ Es indiscutible que tiene efectos tóxicos en los niños. ${ }^{2,3}$

En México se han realizado varios estudios para medir la exposición a plomo en los niños. ${ }^{4-9}$ Los datos indican que, en la última década, ha habido una caída en las concentraciones de plomo sanguíneo que coincide con el control que se ha ejercido sobre algunas fuentes de exposición no ocupacional a dicho metal. ${ }^{10}$ Sin embargo, existen otras fuentes de exposición, como la loza vidriada con plomo, cocida a baja temperatura, que todavía ocasionan niveles significativos de plomo en sangre. ${ }^{10-12}$

En diversos estudios se ha utilizado la medición del coeficiente intelectual $(\mathrm{CI})^{7,12-14}$ con la finalidad de probar el efecto del plomo en el desempeño neuroconductual.

Los resultados indican que los niños con altos niveles de plomo presentan un desempeño más pobre en las pruebas psicométricas, un rendimiento escolar menor y un desarrollo intelectual deficiente en comparación con aquellos que presentan niveles bajos. ${ }^{3,4,7,14}$

La mayor parte de los estudios en México y en el mundo sobre el efecto del plomo en el desarrollo del niño se han enfocado en evaluar el desarrollo intelectual, para lo cual han utilizado pruebas psicométricas. El CI mide diferentes habilidades, pero muchas pruebas de CI están sesgadas culturalmente. Por eso es inadecuado usar pruebas desarrolladas en una cultura, con personas de otra, y pretender, así, medir la misma habilidad en ambas culturas.

Otro problema relacionado con los estudios de los efectos del plomo con base en el CI es la dificultad para plantear el significado que tiene en la vida del niño una disminución de uno, dos o tres puntos de CI.

Existen, sin embargo, estudios en niños que no presentan los problemas mencionados anteriormente. Un conjunto de estudios ha encontrado efectos de la exposición a plomo en medidas antropométricas de los niños. ${ }^{15-17}$ Otro grupo de estudios ha encontrado efectos del plomo sobre la sensibilidad auditiva, el equilibrio y la habilidad motora. ${ }^{18-21}$ La ventaja de usar tales medidas es evidente, ya que no se ven afectadas culturalmente $\mathrm{y}$, a diferencia de los estudios con CI, estas pruebas contribuyen ampliamente a entender cualquier deficiencia que pueda interferir con la ejecución de habilidades adaptativas comunes a la vida diaria. Estas habilidades incluyen correr, atrapar, lanzar, trazar, copiar y hacer caligrafía, entre muchas otras. Por ejemplo, un niño que no puede oír bien o que no puede equilibrarse va a tener una vida muy diferente a la de un niño sano. Por todo lo anterior, los estudios que asocian los niveles de plomo en sangre de los niños y la coordinación visomotora y el equilibrio son importantes. A su vez, este tipo de estudios nos ayudan a conocer las habilidades específicas que están siendo afectadas por el plomo y que explican la reducción en el coeficiente intelectual. Conocer cuáles son las habilidades específicas que se ven afectadas por el 
plomo nos permite diseñar programas de entrenamiento para ayudar a los niños así afectados.

El objetivo de este estudio fue identificar si existe una asociación negativa entre los niveles de plomo en sangre y la coordinación visomotora y el equilibrio de escolares.

\section{Material y métodos}

Se realizó un estudio transversal, con una muestra no probabilística (muestreo por conveniencia), ${ }^{11}$ en niños de ocho de las 13 escuelas de la zona central educativa (Sector 1, Instituto de Educación Pública del Estado de Oaxaca) de la ciudad de Oaxaca.

Se seleccionó a los niños de cuarto año de primaria de 8 a 10 años de edad, mediante un listado por escuela y grupo. De los 664 estudiantes elegibles, durante noviembre y diciembre de 1998, los padres de $464(69 \%)$ estuvieron de acuerdo en participar y firmaron la carta de consentimiento informado aprobada por el Comité de Etica del Instituto Nacional de Salud Pública en México. Solamente 301 niños (45\%) llenaron el cuestionario, y 280 (42\%) llegaron para la toma de sangre. Se excluyó a nueve niños con 11 años cumplidos al momento de las pruebas, otros 14 cuya escuela estaba fuera de la zona escolar central, un niño con incapacidad física y un niño por falta de datos de pérdida del conocimiento por algún accidente, por lo que la muestra quedó constituida por 255 niños que representaron $38 \%$ del grupo total elegible. En un estudio llevado a cabo con estos niños ${ }^{11}$ se encontró que las variables que tuvieron un mayor impacto para predecir concentraciones elevadas de plomo fueron, en orden de importancia, el uso de barro vidriado con plomo, el ingreso familiar, el uso de grasa animal para cocinar los alimentos del niño y la utilización de los remedios caseros.

Se descartó la existencia de alguna patología (variables confusoras) del oído medio, como otitis media, con un examen de otoscopía realizado por personal médico capacitado, utilizando un otoscopio Welch Allyn ${ }^{\circledR}$ y se evaluó la agudeza visual por medio de una tabla optométrica. Cabe mencionar que ningún niño se excluyó del estudio por presentar alguna de estas patologías.

Se aplicaron dos pruebas estandarizadas recomendadas para valorar el sistema visomotor del niño (coordinación de sus movimientos con los ojos y manos): a) la Evaluación de la percepción visual de Frostig (DTVP2), ${ }^{22}$ y b) la subescala equilibrio de la prueba Frostig Movement Skills Test Battery, ${ }^{23}$ para evaluar el equilibrio; cada una de las pruebas fue aplicada por un médico especializado. Se utilizaron las normas establecidas por los autores en los Estados Unidos de América.

La prueba de Evaluación de la percepción visual de Frostig 2 evalúa el rendimiento del niño en tareas exclusivamente de percepción visual que requieren poca o ninguna habilidad motora (cociente de percepción visual con respuesta motriz reducida), y tareas que involucran integración visomotora o conducta motora guiada visualmente y el grado de destreza motora requerido para ejecutarla (cociente de integración visomotora). Asimismo, está diseñada para usarla en niños de 4 a 10 años de edad y ha sido construida para evaluar la habilidad perceptovisual del niño bajo condiciones de respuesta motriz reducida y respuesta motriz realzada, para que puedan compararse fácilmente. La validez y confiabilidad de dicha prueba han sido ampliamente descritas. ${ }^{22}$

Esta prueba consta de ocho subpruebas (posición en el espacio, figura-fondo, cierre visual, constancia de forma, coordinación ojo-mano, copia, relaciones espaciales y velocidad visomotora). Las subpruebas miden habilidades visomotoras así como habilidades visuales diferentes, aunque relacionadas entre sí, y consisten en una serie de ejercicios con la utilización de lápiz y papel para determinar la coordinación visomotora del niño.

Se calificó de acuerdo con el desempeño, la edad y las puntuaciones crudas, éstas se convierten en puntuaciones estándar para dar puntajes. Los puntajes fueron transformados en tres cocientes, los cuales proporcionan las puntuaciones de la percepción visual general (incluye todas las subpruebas), la percepción visual con respuesta motriz reducida (posición en el espacio, figura-fondo, cierre visual y constancia de forma) y la integración visomotora (coordinación ojomano, copia, relaciones espaciales y velocidad visomotora).

La subescala equilibrio de la prueba Frostig Movement Skills Test Battery fue aplicada también por un médico capacitado, y consta de dos pruebas. La primera mide la habilidad del niño para mantener el equilibrio dinámico, para lo cual aquél camina balanceándose sobre una barra de equilibrio de $3 \mathrm{~m}$ de largo. La barra tiene dos anchos: 5 y $3 \mathrm{~cm}$ y está sostenida por dos bases de $12.5 \mathrm{~cm}$ de altura, por $30 \mathrm{~cm}$ de largo. El niño inicia la prueba sobre la parte más ancha, con un pie delante del otro con las manos en la cintura, en la que se mide el número de pasos que el niño da sin caerse y sin quitar las manos de la cintura. Posteriormente, se repiten las mismas mediciones en el lado más angosto.

En la segunda prueba, con un cronómetro profesional Sports ${ }^{\circledR}$, se cuantificó el tiempo que el niño 
logra mantenerse equilibrado en el piso con un pie y el otro pie descansa en la rodilla, sin tener ningún apoyo, desde el inicio hasta la primera caída con una extremidad, primero con ojos abiertos y luego con ojos cerrados.

Ojos abiertos: puede ocupar sus órganos vestibulares y canales semicirculares y órgano de otolito. Además para orientarse utiliza la función visual.

Ojos cerrados: se eliminó el uso de retroalimentación visual y se midieron factores vestíbulo-otolito en vez de combinación vestibular-otolito y visual.

En cuanto al monitoreo biológico se cuantificó concentración de plomo en sangre. Se tomó una muestra de $5 \mathrm{ml}$ de sangre venosa posterior a una limpieza completa en el sitio de punción. Se emplearon tubos de sistema al vacío con tapa azul (libre de metales) con EDTA (sal sódica de ácido etilén-amino-tetra azuoico), o tapa morada con heparina. No hubo diferencias importantes en el nivel de plomo en ambos tubos. Las muestras se refrigeraron a una temperatura de $4{ }^{\circ} \mathrm{C}$ hasta el envío al Laboratorio de Toxicología, de la Universidad de Drew, en Los Angeles, California, Estados Unidos de América (EUA). Se analizaron las muestras por duplicado por el método de espectrometría de absorción atómica con horno de grafito, ${ }^{24}$ utilizando el promedio de las dos mediciones.

El laboratorio cumple con el Programa de Control de Calidad de los Centros para la Prevención y el Control de Enfermedades de Atlanta, Georgia, y del College of American Pathologists, de EUA, para medir plomo en sangre. Durante el análisis una de cada cinco muestras es una muestra estándar.

Para conocer si ha existido exposición a plomo por diferentes fuentes se aplicó un cuestionario validado en otros estudios ${ }^{5,11}$-con una entrevista estructurada a las madres-, el cual evalúa variables tales como sexo, edad, nivel socioeconómico, educación escolar de la madre y del padre e ingreso, entre otras. El cuestionario también evaluó historia de salud del niño e información para conocer los antecedentes que pudieran interferir con su desempeño en las pruebas, como otitis media.

Los datos fueron capturados por duplicado y verificados con una base de datos en Foxpro. La verificación consistió en la revisión de los rangos de cada una de las variables, las inconsistencias y los valores faltantes. En el análisis estadístico se valoró la relación entre las variables independientes y la dependiente mediante un programa estadístico de análisis de datos, el Stata versión 6.0 (Stata Corporation, College Station, Texas, EUA).
La exploración de los datos se realizó mediante estadísticas descriptivas y gráficas para identificar valores extremos, minimizar los errores y encontrar sus distribuciones. Se hizo la revisión total de la base de datos y se compararon con los datos originales para verificar su confiabilidad.

Se usaron pruebas de $\chi^{2}, t$ de Student, y $U$ de Mann-Whitney para grupos independientes, con el fin de determinar si las familias cuyas madres de los niños que no habían llenado el cuestionario eran diferentes de los participantes con datos completos.

Antes de construir modelos multivariados de las variables dependientes, se exploró la relación univariada y bivariada entre las variables dependientes y de control. Se seleccionaron aquellas variables de control que mostraron una asociación significativa al nivel de probabilidad menor de 0.10 para su uso en los modelos multivariados. Las variables seleccionadas fueron: nivel de plomo en sangre; ingreso familiar (ordinales); sexo (M/F); datos de pérdida del conocimiento por algún accidente (sí/no); edad en meses (continua), y el cociente de integración visomotora de las pruebas de Frostig, promedio de las puntuaciones en las subpruebas coordinación ojo-mano y relaciones espaciales. Estas variables se utilizaron en todos los modelos probados.

La distribución de los niveles de plomo en sangre se dividió en tres grupos, el primero incluyó los niveles de plomo en un rango de 0-9.9 $\mu \mathrm{g} / \mathrm{dl}$; el segundo, en un rango de 10.0-19.9 $\mu \mathrm{g} / \mathrm{dl}$, y el tercero, los niveles mayores de $20 \mu \mathrm{g} / \mathrm{dl}$.

El análisis multivariado se llevó a cabo mediante una regresión lineal múltiple, ya que la variable de respuesta presentaba valores continuos. El diagnóstico de los modelos se realizó por medio de pruebas de heteroscedasticidad y de normalidad de los residuales y mediante gráficas de distribución de los mismos.

La regresión lineal múltiple se aplicó al cociente de integración visomotora de la prueba de Frostig, al igual que para la prueba de equilibrio (tiempo de las caídas). La regresión ordinal múltiple se utilizó para probar las subpruebas de Frostig de integración visomotora y para probar la prueba de equilibrio (en número de pasos).

Se probó el efecto del plomo dentro del modelo al utilizar una técnica de simulaciones de Montecarlo, ${ }^{25,26}$ estimado por 1000 simulaciones. Se obtuvo mil veces una submuestra de los datos haciendo los análisis cada vez, con el mismo modelo se calcularon los promedios y sus respectivos límites de confianza al $90 \%$, de este conjunto de simulaciones con el cociente de integración visomotora. 


\section{Resultados}

En la población de 255 niños se obtuvo una media geométrica de concentraciones de plomo en sangre de $11.5 \mu \mathrm{g} / \mathrm{dl}$ (DE geométrica $=+6.3,-5.2)$.

El cuadro I muestra las características descriptivas de los niños, según las diferentes variables predictoras mencionadas anteriormente.
No hubo diferencias importantes entre grupos sobre cualquiera de las variables que se usó en los análisis de regresión $(p>0.10)$. Los niños excluidos de la muestra eran significativamente más jóvenes (9.0 años, $p=0.03$ ) que los incluidos (9.1 años).

De acuerdo con el análisis multivariado el cociente de integración visomotora resultó significativamente relacionado con el plomo ( $p>0.042)$ (cuadro II). Para

\section{Cuadro I \\ Características descriptivas de los NiÑos, SEgún diferentes Variables PRedictoras de nivel de plomo en SANGre. OAXaca, México, 1998}

\begin{tabular}{|c|c|c|c|c|}
\hline Variables & $n$ & $\bar{x}$ & DE* & Rango \\
\hline N ivel de plomo sanguíneo ( $\mu \mathrm{g} / \mathrm{dl})$ & 255 & 11.5 & $6.3,-5.2$ & $1.30-35.50$ \\
\hline $0-9.9$ & 114 & & & \\
\hline $10-19.9$ & 116 & & & \\
\hline$>20$ & 25 & & & \\
\hline Sexo (femenino) & $134(52.6 \%)$ & & & \\
\hline Pérdida del conocimiento ( $\mathrm{N}$ o) & $243(95.3 \%)$ & & & \\
\hline Ingreso per cápita (sueldos mínimos) & 255 & 1.41 & 0.893 & $0.2-5$ \\
\hline Edad (meses) & 255 & 114.09 & 5.04 & $96-131$ \\
\hline \multicolumn{5}{|l|}{ Pruebas de la percepción visual de Frostig } \\
\hline PVG & 255 & 96.72 & 10.02 & $72-125$ \\
\hline PVRMR & 255 & 89.10 & 13.44 & $62-128$ \\
\hline IV & 255 & 104.55 & 9.38 & $77-128$ \\
\hline \multicolumn{5}{|l|}{ Pruebas de equilibrio } \\
\hline Barra (número de pasos) & 255 & 8.92 & 3.96 & $0-16$ \\
\hline Tiempo (ojos abiertos) & 255 & 12.12 & 7.71 & $0-20$ \\
\hline Tiempo (ojos cerrados) & 255 & 8.62 & 2.63 & $1-20$ \\
\hline
\end{tabular}

*DE: desviación estándar geométrica y media geométrica sólo para plomo

X: media

PVG : percepción visual general

PVRMR: percepción visual con respuesta motriz reducida

IV: integración visomotora

\section{Cuadro II}

\section{Distribución del COCIENTE Y SUbPRUEbas de INTEgRACIÓN VISOMOTORA DE LA PRUEba DE EVALUACIÓN de la percepción visual de Frostig y nivel de plomo en sangre. OaxaCa, México, 1998}

\begin{tabular}{|c|c|c|c|c|}
\hline & & jrupos de plomo en sangre & & \\
\hline & $0-9.9 \mu \mathrm{g} / \mathrm{dl} \mathrm{n}=114$ & $10.0-19.9 \mu \mathrm{g} / \mathrm{d} \mid \mathrm{n}=116$ & $>20 \mu \mathrm{g} / \mathrm{dl} \mathrm{n}=25$ & Probabilidad* \\
\hline Cociente & & & & \\
\hline Integración visomotora (datos medidos) & 105.75 & 104.50 & 99.28 & 0.040 \\
\hline Integración visomotora (modelo simulado ${ }^{\ddagger}$ ) & 105.73 & 103.93 & 102.12 & 0.042 \\
\hline Subpruebas & & & & \\
\hline Coordinación ojo-mano (datos medidos) & 10.34 & 10.23 & 7.96 & 0.045 \\
\hline Relaciones espaciales (datos medidos) & 12.27 & 11.78 & 11.52 & 0.039 \\
\hline
\end{tabular}

* N ivel de significancia de la tendencia lineal de los cocientes con aumento de plomo sanguíneo

₹ 1000 simulaciones de Montecarlo, ajustadas por las variables del modelo 
determinar cuáles subpruebas de este cociente contribuyeron más a la relación con plomo se analizaron las subpruebas de esta escala. Sólo la coordinación ojomano y las relaciones espaciales resultaron significativamente relacionadas con el plomo $(p=0.045$ y $p=0.039$, respectivamente)(cuadro II). Se realizaron análisis multivariados con las subpruebas de copia, velocidad visomotora y cociente de respuesta motriz reducida, sin embargo, los coeficientes no resultaron significativos.

En el análisis multivariado (cuadro III) se observó una relación significativa entre los niveles de plomo en sangre y el cociente de integración visomotora, también se observó que éste disminuye 1.78 (IC 95\% $-3.51,-0.06)$ puntos por cada $10.0 \mu \mathrm{g} / \mathrm{dl}$ de aumento en los niveles de plomo en sangre del niño. De las subpruebas que resultaron significativas se observó una disminución de 0.34 (IC 95\% -0.681, -0.008) puntos en la coordinación ojo-mano, y 0.37 (IC 95\% -0.721, -0.019) puntos en las relaciones espaciales por cada $10 \mu \mathrm{g} / \mathrm{dl}$ de aumento en los niveles de plomo en sangre del niño, ajustando por sexo, edad en meses, ingreso y antecedentes de pérdida del conocimiento en cada uno de los modelos. Es importante señalar que el nivel de ingreso económico resultó significativo debido a que a mayor ingreso, los resultados de las pruebas son más altos (cuadro IV).

En la figura 1 se muestran los cocientes ajustados por el modelo de la integración visomotora de la prueba de la evaluación de la percepción visual de Frostig, con categoría de plomo sanguíneo del niño, donde las líneas verticales indican los errores estándar. El cociente de integración visomotora asociado con la categoría de plomo en sangre mayor de $20 \mu \mathrm{g} / \mathrm{dl}$ es significativamente más bajo que el cociente asociado con la categoría de plomo menor de $10 \mu \mathrm{g} / \mathrm{dl}(p>0.05)$ (cuadro II).

Se repitió el análisis con el mismo modelo, utilizando plomo como una variable continua y en una regresión lowess para mostrar la no linealidad de la

\section{Cuadro III \\ Modelo de Regresión lineal múltiple de LA PRUeba de eVAluación de LA PeRCEPCión Visual de Frostig del cociente de integración visomotora. OAXaca, México, 1998}

$\begin{array}{lccrr}\text { Variables } & \text { Coeficiente } & \text { Error estándar } & \text { Valor de } p & \text { IC } 95 \% \\ & & & & \\ \text { Nivel de plomo sanguíneo }(\mu \mathrm{g} / \mathrm{d}) \text { )* } & -1.788 & 0.874 & 0.042 & -3.510-0.065 \\ \text { Sexo (femenino) } & 2.555 & 1.127 & 0.024 & 0.3334 .776 \\ \text { Pérdida del conocimiento (N o) } & 7.139 & 2.702 & 0.009 & 1.81812 .461 \\ \text { Ingreso per cápita (sueldos mínimos) } & 1.345 & 0.641 & 0.037 & 0.0822 .607 \\ \text { Edad (meses) } & -0.169 & 0.112 & 0.132 & -0.3890 .051\end{array}$

$n=255$

$R^{2}=0.108$

IC 95\%: intervalo de confianza al 95\%

*Variable ordinal con tres niveles de plomo en sangre 0-9.9, 10-19.9 y >20 en $\mu \mathrm{g} / \mathrm{dl}$

\section{Cuadro IV \\ MOdeLOS DE REGRESIÓN ORDINAL MÚLTIPLE DE LAS SUBPRUEBAS DE COORDINACIÓN OJO-MANO Y RELACIONES ESPACIALES QUE CONTRIBUYEN AL COCIENTE DE INTEGRACIÓN VISOMOTORA Y Relacionados significativamente con plomo. OaXaca, México, 1998}

\begin{tabular}{|c|c|c|c|c|c|c|c|c|}
\hline \multirow[b]{2}{*}{ Variables } & \multicolumn{4}{|c|}{ Coordinación ojo-mano } & \multicolumn{4}{|c|}{ Relaciones espaciales } \\
\hline & Coeficiente & ES & Valor de $p$ & IC $95 \%$ & Coeficiente & ES & Valor de $p$ & IC 95\% \\
\hline $\mathrm{N}$ ivel de plomo sanguíneo $(\mu \mathrm{g} / \mathrm{d}) *$ & -0.344 & 0.178 & 0.045 & $-0.681-0.007$ & -0.369 & 0.178 & 0.039 & $-0.720-0.019$ \\
\hline Sexo (femenino) & 0.100 & 0.225 & 0.656 & -0.3410 .542 & 0.398 & 0.251 & 0.113 & -0.0940 .891 \\
\hline Pérdida del conocimiento ( $\mathrm{N} 0$ ) & 0.828 & 0.385 & 0.032 & 0.0731 .584 & 0.048 & 0.647 & 0.940 & -1.2211 .318 \\
\hline Ingreso per cápita (sueldos mínimos) & 0.285 & 0.114 & 0.013 & 0.0600 .510 & 0.137 & 0.134 & 0.307 & -0.1260 .402 \\
\hline Edad (meses) & -0.039 & 0.019 & 0.048 & $-0.078-0.000$ & -0.029 & 0.025 & 0.244 & -0.0780 .019 \\
\hline$n=255$ & & g likelif & $0 d=-600.5$ & & & og like & $0 d=-358$ & \\
\hline
\end{tabular}

ES: error estándar

IC 95\%: intervalo de confianza al $95 \%$

* Variable ordinal con tres niveles de plomo en sangre 0-9.9, 10-19.9 y >20 en $\mu \mathrm{g} / \mathrm{dl}$ 


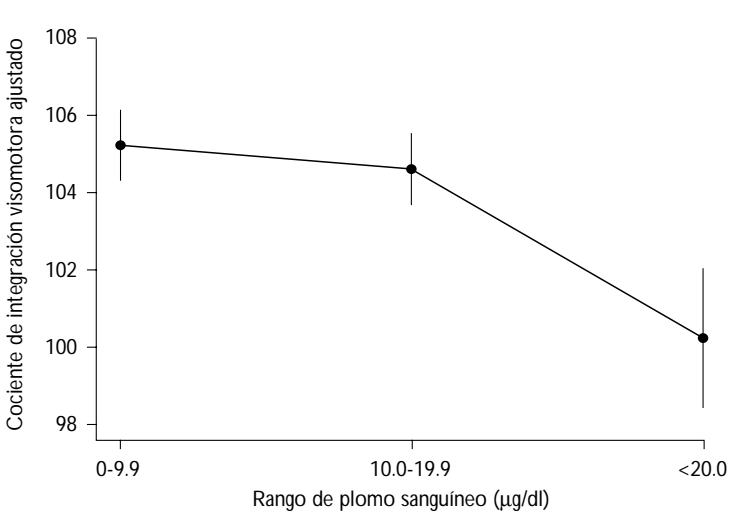

N ota: Las barras de error alrededor de cada punto representan los errores estándar

Figura 1. Cambio del cociente de integración visoMOTORA DE LA PRUEBa de LA EVALUACIÓN DE LA PERCEPCIÓN VISUAL de Frostig (DTVP2), CON CATEgoría de plomo sanguíneo del niño. OAXaCa, México, 1998

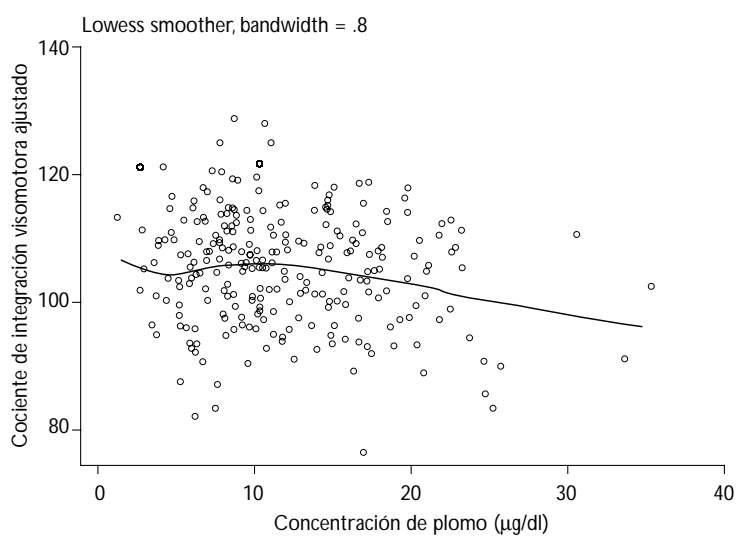

Nota: Se observa la no linealidad del efecto del plomo, mismo que empieza entre 10 y $20 \mu \mathrm{g} / \mathrm{dl}$, razón por la cual se usó la variable ordinal de plomo en el modelo

Figura 2. Regresión lowess de la relación entre el PLOMO Y EL COCIENTE DE INTEGRACIÓN VISOMOTORA AJUSTADO POR LAS OTRAS VARIABLES EN EL MODELO. OAXACA, MÉxico, 1998

relación entre el plomo y el cociente de integración visomotora (figura 2).

No encontramos una relación significativa entre el plomo y el cociente de la respuesta motriz reducida ni en el cociente de percepción visual general. Tampoco se encontró una relación significativa entre el plomo y las pruebas de equilibrio (análisis no mostrados).

\section{Discusión}

Existen estudios que muestran que el plomo ocasiona diferentes efectos en el organismo, de los cuales los que más se han estudiado son los relacionados con el desarrollo intelectual. ${ }^{4}$ Sin embargo, se han realizado enormes esfuerzos en la década pasada para caracterizar los efectos del plomo sobre el desarrollo del organismo. ${ }^{27}$ Por estas razones es importante realizar estudios que nos permitan evaluar los daños a nivel de integración visomotora.

Dado que en México no existen estudios donde se evalúen las pruebas de la percepción visual de Frostig (DTVP2) con un diseño similar al presente estudio, el único punto de comparación son los estudios realizados en otros países, en los que los niños con altos niveles de plomo ( $>20 \mu \mathrm{g} / \mathrm{dl})$ tuvieron peor integración visomotora y tiempo de reacción serial que los niños con bajos niveles de plomo (promedio de $5 \mu \mathrm{g} / \mathrm{dl}$ ). ${ }^{19}$ Otro estudio transversal en Europa encontró resultados similares.$^{28}$ Los resultados del presente estudio coinciden en parte con los obtenidos en esos estudios realizados en niños con niveles similares de plomo en sangre, en cuanto a que el plomo afecta sus habilidades visomotoras.

Los resultados de estudios longitudinales también muestran coincidencia con los obtenidos en este trabajo. El estudio prospectivo de Cincinnati ${ }^{29,30}$ reportó una relación inversa entre el plomo sanguíneo posnatal y la integración visomotora y funcionamiento visoespacial en niños. El estudio prospectivo en Port Pirie, en Australia, ${ }^{18}$ encontró una relación inversa entre los niveles de plomo posnatales hasta los 84 meses de edad y la integración visomotora a los siete años.

Para tener una referencia del nivel socioeconómico se obtuvieron datos mediante la variable de ingreso económico, los resultados coinciden con otros autores $^{29}$ que han mencionado que los niños con déficit de integración visomotora y tiempo de reacción son los niños socialmente afectados, si se les compara con los de clase media.

$\mathrm{Si}$ los efectos medidos en la muestra fueran permanentes podríamos hipotetizar que el plomo, en el rango medido, puede afectar las habilidades para desempeñar tareas que requieran movimientos coordinados con sus manos y ojos, al igual que actividades diarias como deportes, manualidades u oficios.

En el cociente de integración visomotora 69 niños tuvieron puntuaciones menores de 100 (promedio estándar), pero sólo 3.1\% de ellos se encuentra con puntuaciones clínicamente anormales (por debajo de 85). El porcentaje de niños cuyas subpruebas presentaron puntuaciones por debajo de siete (10 es el estándar 
considerado como normal por los autores), fue de $11 \%$ en la subprueba de coordinación ojo-mano y $4.3 \%$ en la subprueba de relaciones espaciales.

En el presente estudio no encontramos una relación significativa entre el nivel de plomo en sangre y el equilibrio como han encontrado otros autores. ${ }^{20,21}$ Esto se puede explicar por el hecho de que un estudio transversal, como éste, carece de la oportunidad de medir la historia de la exposición, los niños participantes diferían en cuanto a edad de los evaluados en otros estudios y la medición del equilibrio utilizada en estudios previos era más precisa y sensible a cambios motores que reflejan el autoajuste ante el desequilibrio que las mediciones usadas en éste.

A pesar de que el efecto del plomo en este estudio resulta significativo, en el rango de concentración menor de $20 \mu \mathrm{g} / \mathrm{dl}$ su efecto fue leve. Hay que recordar que la medición de plomo se llevó a cabo sólo una vez al momento de aplicar las pruebas, por lo que no forzosamente representa la exposición en el pasado, y que las pruebas utilizadas fueron muy sencillas. En el archivo de datos existe una variable que probablemente refleje la exposición al plomo de más larga duración, el uso de barro vidriado con plomo en la casa. Se probó el efecto de usar barro vidriado en el cociente de integración visomotor con una prueba de $t$ para muestras de varianzas no iguales. Los no usuarios obtuvieron una media de 106.5 y los usuarios una media de 103.6, la diferencia entre los grupos fue significativa $(p=$ 0.009). Estos últimos resultados refuerzan la necesidad de eliminar esta fuente de contaminación.

\section{Conclusiones}

Este estudio es otro ejemplo de que el sistema visomotor (evaluado por el cociente de la integración visomotora, y las subpruebas de coordinación ojo-mano y relaciones espaciales) se ve afectado por la exposición a plomo de los niños. Aun cuando el efecto es sutil, resulta indicativo de que incluso a concentraciones consideradas como asintomáticas el plomo es dañino para el organismo.

Sólo $3.1 \%$ de los niños obtuvieron puntuaciones clínicamente anormales, por lo que no podemos decir con certeza que un niño expuesto a niveles de plomo en sangre superiores a $20 \mu \mathrm{g} / \mathrm{dl}$ va a tener un trastorno grave (clínicamente anormal) en las habilidades visomotoras. Sin embargo, aun cuando el impacto de concentraciones de plomo superiores a $20 \mu \mathrm{g} / \mathrm{dl}$ en su desarrollo visomotor puede no ser detectable clínicamente en un niño en particular, sí puede ser significativo en toda una población infantil, que pueda ver limitada su capacidad para incorporarse a un mejor trabajo, oficio o profesión que le brinde un mejor estilo de vida.

Los resultados de este estudio señalan la necesidad de reforzar la iniciativa para reducir la exposición de los niños a fuentes de plomo conocidas. En esta población de estudio la cerámica vidriada tradicional es la principal fuente asociada a concentraciones elevadas de plomo en sangre. ${ }^{11}$

Para prevenir daños a la población sería importante impulsar acciones preventivas enfocadas en la modificación de conductas que pueden incrementar el riesgo de exposición a plomo. Para lograr un mayor impacto poblacional el paso más importante sería la eliminación del uso de barro vidriado con plomo.

Además, tomar medidas como acciones en educación a la población y proponer soluciones para disminuir la exposición a plomo, ya conocidas, involucrando a los organismos gubernamentales, industrias e instituciones educativas, así como a los medios de comunicación y a los padres de familia, pueden reducir las fuentes de exposición con las que la población está en contacto.

Asimismo, cuando hay un alto grado de exposición en un individuo hay que tratarlo individualmente, aplicando medidas preventivas.

\section{Agradecimientos}

Un especial agradecimiento por la colaboración de los niños y padres de familia, al supervisor de Zona 1, directores y maestros de las ocho escuelas que participaron y a las autoridades del sistema educativo del Instituto de Educación Pública del Estado de Oaxaca por las facilidades brindadas para la realización de este estudio; también al personal del Laboratorio de Toxicología, de la Universidad de Drew, en Los Angeles, California, y a las psicólogas del Instituto Nacional de Perinatología de México: Sandra Martínez, Carmen Hernández, Erika Osorio y María Eugenia Wong por su ayuda profesional.

Asimismo, se agradece ampliamente la contribución profesional de la M. en C. Leticia Hernández Cadena, del doctor Arturo C. Juárez Pérez y de la M. en C. Guadalupe Aguilar Madrid, del Instituto Nacional de Salud Pública; a Jorge López por su apoyo como capturista y al personal que participó en el trabajo de campo.

\section{Referencias}

1. Banks E, Ferreti L, Shucard D. Effects of low level lead exposure on cognitive function in children:A review of behavioral, neuropsychological and biological evidence. N eurotoxicology 1997;18(1):237-282. 
2. Verberk $M, W$ illems T,Verplanke $A, W$ olff F. Environmental lead and renal effects in children. Arch Environ Health 1996;51(1):83-87.

3. Gulson B, Davis J, Mizon K, Kotsch M, Law A, Howarth D. Lead bioavailability in the environment of children: Blood lead levels in children can be elevated in a mining community.Arch Environ Health 1994;49(5):326-331. 4. Hérnandez M, Palazuelos E. Intoxicación por plomo en México: prevención y control. Cuernavaca, Morelos: Instituto N acional de Salud Pública (Perspectivas en Salud Pública núm. 21), 1995:21-61.

5. Jiménez C, Romieu I, Palazuelos E. Factores de exposición ambiental y concentraciones de plomo en sangre en niños de la ciudad de México. Salud Publica Mex 1993;35:599-605.

6. Intoxicación por plomo: de la detección a la prevención primaria (trad.). Salud Publica Mex 1995;37(3):264-276.

7. Muñoz H, Romieu I, Palazuelos E. Blood lead level and neurobehavioral development amog children living in Mexico City. Arch Environ Health 1993:48:132-138.

8. Romieu I, Palazuelos E, Meneses F, Hernández-Avila M.Vehicular traffic as a determinant of children blood lead levels: A pilot study in Mexico City. Arch Environ Health 1992;47(4):246-249.

9. Romieu I, Carreon T, Lopez L, Palazuelos E. Enviromental urban lead exposure and blood lead levels in children of Mexico City. Environ Health Perspect 1995;103:2-6.

10. Rothenberg SJ, Schnaas L, Perroni E, Hernández RM, Karchmer S. Secular trend in blood lead levels in a cohort of Mexico City children.Arch Environ Health 1998;53(3):231-235.

11. Azcona MI, Rothenberg SJ, Schnaas L, Zamora JS, Romero M. Leadglazed ceramic ware and blood lead levels of children in the $C$ ity of 0 axaca, Mexico.Arch Environ Health 2000;55(3). En prensa.

12. Calderón $S$, Valdez $B$, Mazúniga $C$. Lead exposure in a population of Mexican children. Hum Exp Toxicol 1996;15.

13. Molina D, Zúñiga $M, C$ árdenas $A$. Alteraciones psicológicas en niños expuestos a ambientes domésticos ricos en plomo. Bol 0 ficina Sanit Panam 1983;94:3.

14. Schnaas L, Rothenberg SJ, Perroni E, Hernández RM, Hérnandez C, Martínez S. Relación entre la exposición prenatal y postnatal al plomo y el desarrollo intelectual del niño a los 42 meses de edad. Perinatol Reprod Hum 1999;13(3):214-220.

15. Shukla R, Bornschein R, D ietrich K, Buncher $C$, Berger 0 , Hammond $P$ et al. Fetal and infant lead exposure: Effects on growth in stature. Pediatrics 1989:84:604-612.

16. Rothenberg SJ, Schnaas L, Perroni E, Hérnandez R. Pre and postnatal lead effect on head circumference: A case for critical periods. N eurotoxicol Teratol 1999;21:1-11.

17. Schnaas L, Rothenberg SJ, Perroni E, Hernández RM, Hérnandez C, Martínez S. Relación entre la exposición prenatal al plomo y la talla del niño a los 3 años de edad. Perinatol Reprod Hum 1998;12(3):151-156.
18. Baghurst A, McMichael J, Shilu T. Exposure to environmental lead and visual-motor integration at age 7 years:The Port Pirie Cohort Study. Epidemiology 1995;6:104-109.

19.W inneke G, BrockhausA, Ewers U, N euf M. Results from the European multicenter study on lead neurotoxicity in children: Implications for risk assessment. N eurotoxicol Teratol 1990;12:553-559.

20. Bhattacharya A, Shukla R, Bornschein K. Lead effects on postural balance of children. Environ Health Perspect 1990;89:35-42.

21. Bhattacharya A, Shukla R, Bornschein K. Postural disequilibrium quantification in children with chronic lead exposure:A pilot study. N eurotoxicology 1988;3:327-340.

22. Hammill D. Evaluación de la percepción visual de Frostig (DTVP-2) México, D.F.: Manual Moderno, 1995.

23. O rpet A. Frostig Movement Skills Test Battery. Palo Alto (CA): Consulting Psychologists Press, Inc., 1972.

24. $\mathrm{N}$ ational Institute for $\mathrm{O}$ ccupational Safety and $\mathrm{Health}$. Manual of analytical methods. Eller PM. 3a. edición. Cincinnati (O H): N IO SH, 1984, and 1990;M ethod N 0s. 7082, 7105 Suppl.

25. Tomz M, W ittenberg J, King G. Clarify: Software for interpreting and presenting statistical results. Version 1.2. Cambridge (MA): Harvard University, September 16, 1998.

26. King G, Tomz M, W ittenberg J. Making the most of statistical analyses: Improving interpretation and presentation. Paper prepared for presentation at the Annual Meetings of the A merican Political Science Association; 1998 August. Boston (MA), EUA.

27. Rice DC. Behavioral effects of lead: Commonalities between experimental and epidemiologic data. Environ Health Perspect 1996;104(2): 337-351.

28. Hansen 0 , Trillingsgaard A, Beese I, Lyngbye T. A neuropsychological study of children with elevated dentine lead level:Assessment of the effect of lead in different socio-economic groups. Neurotoxicol Teratol 1989:11:205-213.

29. Dietrich KN, Succop P, Berger O. Lead exposure and the cognitive development of urban preschool children: The Cincinnati lead study cohort at age 4 years. N eurotoxicol Teratol 1991;13:203-211.

30. Dietrich KN , Succop P, Berger O. Lead exposure and the motor developmental status of urban six-year-old children in the $\mathrm{C}$ incinnati prospective study. Pediatrics 1993; 91:301-307.

31.W inneke G, Kraemer U. N europsychological effects of lead in children: Interactions with social background variables. Neuropsychobiology 1984;11:195-202. 